Pacific Journal of Mathematics

A MINIMAL UPPER BOUND ON A SEQUENCE OF TURING 


\title{
A MINIMAL UPPER BOUND ON A SEQUENCE OF TURING DEGREES WHICH REPRESENTS THAT SEQUENCE
}

\author{
HAROLD T. HODES
}

\begin{abstract}
Given a sequence of Turing degrees $\left\langle a_{i}\right\rangle_{i<\omega}, a_{i}<a_{i+1}$, is there a function of $f$ such that (i) $\operatorname{deg}(f)$ is a minimal upper bound on $\left\langle a_{i}\right\rangle_{i<\omega}$, and (ii) $\left\{\operatorname{deg}\left((f)_{n}\right) \mid n<\omega\right\}=\left\{a_{i} \mid i<\omega\right\}$ ? In this note we show that the most natural minimal upper bound on $\left\langle a_{1}\right\rangle_{i<\omega}$ is of the form $\operatorname{deg}(f)$ for such an $f$.
\end{abstract}

Because there seem to be a cluster of interesting notions and question related to this problem, we start with some definitions. Fix a recursive pairing function $(x, y) \mapsto\langle x, y\rangle ;(f)_{x}(y)=f(\langle x, y\rangle)$. Where $I$ is a set of Turing degrees and $f \in{ }^{\omega} \omega, f$ represents (subrepresents) $I$ iff $I=$ $\left\{\operatorname{deg}\left((f)_{n}\right) \mid n<\omega\right\}\left(I \subseteq\left\{\operatorname{deg}\left((f)_{n}\right) \mid n<\omega\right\}\right)$. For $I^{\prime} \subseteq I, I^{\prime}$ is cofinal in $I$ iff for every $a \in I$ there is a $b \in I^{\prime}$ with $a \leq b$. $f$ weakly represents (weakly subrepresents) $I$ iff $f$ represents (subrepresents) some $I^{\prime}$ cofinal in I. A degree a represents (subrepresents, weakly represents, weakly subrepresents) $I$ iff some $f \in$ a does so. $I$ is an ideal iff $I$ is non-empty closed downward and under join.

Terminology. A tree $T$ is a total function from $2^{<\omega}=$ Str into Str so that for any $\delta \in \operatorname{Str}, T\left(\delta^{\wedge} 0\right)$ and $T(\delta \hat{~} 1)$ are incompatible extensions of $T(\delta) . \delta \in \operatorname{Str}(s)$ iff $\delta \in \operatorname{Str}$ and $\operatorname{dom}(\delta)=s$. A pre-tree of height $s$ is a function $T: \operatorname{Str}(s) \rightarrow \operatorname{Str}$ where for all $\delta \in \operatorname{Str}(s-1), T\left(\delta^{\wedge}\langle 0\rangle\right)$ and $T\left(\delta^{\wedge}\langle 1\rangle\right)$ are incompatible extensions of $T(\delta)$. For $\delta \in \operatorname{Str}$ and $A \in{ }^{\omega} 2$, $\delta \subseteq A$ iff for all $i \in \operatorname{dom}(\delta), \delta(i)=A(i)$. Where $T$ is a tree, $B \in[T]$ iff for some $A \in{ }^{\omega} 2$; for all $n, T(A \uparrow n) \subset B$; (i.e. $B$ is a path through $T$ ). Where $T$ is a pre-tree of height $s, B \in[T]$ iff for some $\delta \in \operatorname{Str}, \operatorname{dom}(\delta)=s$ and $T(\delta) \subset B$.

Where $T$ is a tree and $A \in{ }^{\omega} 2$, let

$$
\operatorname{Code}(T, A)(\delta)=T(\langle A(0), \delta(0), \ldots, \delta(n-1), A(n)\rangle),
$$

where $n=\operatorname{dom}(\delta)-1$. Notice: $\operatorname{Code}(T, A)(\langle\rangle) \supsetneqq T(\langle\rangle)$. Where $T$ is a pre-tree of height $\leq 2 n+1$ and $\tau \in \operatorname{Str}, \operatorname{dom}(\tau) \geq n, \operatorname{Code}(T, \tau)$ is defined similarly. For $T$ a tree (pre-tree) and $B \in[T]$, let $\operatorname{Coded}(B, T)$ be the real $A \in{ }^{\omega} 2$ (string $\tau$ ) such that $A(e)=i(\tau(e)=i$ ) iff for some $\delta$, $T(\delta) \subseteq B$ and $\delta(2 e)=i$. If $T$ is a pre-tree of height $2 n$ or $2 n+1$, 
$\operatorname{dom}(\operatorname{Coded}(B, T))=n$; so if $T$ is a pre-tree, $B \in[T]$ and $\tau=$ $\operatorname{Coded}(B, T), \operatorname{Code}(T, \tau)$ is well defined.

We'll say that $\tau$ is on $T$ iff $\tau \in \operatorname{Range}(T)$. Let $\tau_{0}, \tau_{1}$ be an $e$-splitting of $\tau$ iff $\tau_{0}, \tau_{1} \supseteq \tau$ and for some $x$ and $t,\{e\}_{t}^{\tau_{0}}(x)$ and $\{e\}_{t}^{\tau_{1}}(x)$ are defined and different. By "the least $e$-splitting of $\tau$ ", we mean that $\left\langle\tau_{0}, \tau_{1}, x, t\right\rangle$ is minimal. Where $T$ is a tree, let $e-\operatorname{Split}(T)(\langle\rangle)=\mathrm{T}(\langle\rangle)$; if $e-\operatorname{Split}(T)(\delta)$ is defined, $e$-Split $(T)\left(\delta^{\wedge}\langle 0\rangle\right), e-\operatorname{Split}(T)\left(\delta^{\wedge}\langle 1\rangle\right)$ is the least $e$-splitting of $e-\operatorname{Split}(T)(\delta)$ on $T$, if such there be; otherwise they are undefined. Clearly $e-\operatorname{Split}(T)$ is partial-recursive in $T$.

Where $T$ is a pre-tree, $e$-Split ${ }_{s}(T)$ is defined like $e-\operatorname{Split}(T)$, except that (1) all searches for $e$-splittings on $T$ are bounded by $s ;(2) e$-Split $(T)(\delta)$ is defined iff for all $\tau$ with $\operatorname{dom}(\tau)=\operatorname{dom}(\delta), e-\operatorname{Split}(T)(\tau)$ is defined. (2) insures that $e$-Split ${ }_{s}(T)$ is a pre-tree. For $T$ a tree or pre-tree, $\operatorname{Full}(T, \delta)(\tau)$ $=T(\hat{\delta} \tau)$. (If $\delta \notin \operatorname{dom}(T), \operatorname{Full}(T, \delta)=\varnothing$, which is still a pre-tree.)

Theorem. Suppose $I=\left\{\mathbf{a}_{i} \mid i<\omega\right\}$ is a sequence of Turing degrees, and for all $i, \mathbf{a}_{i}<\mathbf{a}_{i+1}$. Then some minimal upper-bound on $I$ represents $I$.

To prove this, we use the simplest construction of a minimal upper bound on $I$. Fix $\left\langle A_{i}\right\rangle_{i<\omega}$ so that for all $i, A_{i} \in \mathbf{a}_{i}$. Let $T_{-1}=\operatorname{Id} \uparrow$ Str.

$$
T_{2 e}= \begin{cases}e-\operatorname{Split}\left(T_{2 e-1}\right) & \text { if } e-\operatorname{Split}\left(T_{2 e-1}\right) \text { is total } \\ \operatorname{Full}\left(T_{2 e-1}, \tau_{e}\right) & \text { otherwise }\end{cases}
$$

where $\tau_{e}$ is the least $\tau$ such that $T_{2 e-1}(\tau)$ is on $e-\operatorname{Split}\left(T_{2 e-1}\right)(\tau)$ and has no $e$-splitting on $T_{2 e-1}$.

$$
T_{2 e+1}=\operatorname{Code}\left(T_{2 e}, A_{e}\right) .
$$

A tree $T$ is uniformly recursively pointed iff for some $e, T=\{e\}^{B}$ for all $B \in[T]$. All $T_{e}$ are uniformly recursively pointed, and so $T_{2 e-1} \equiv_{T} T_{2 e} \leq_{T} T_{2 e+1} \leq_{T} A_{e}$. Let $\{B\}=\bigcap_{e<\omega}\left[T_{e}\right]$; where $\mathbf{b}=\operatorname{deg}(B), \mathbf{b}$ is a minimal upper bound on $I$. We must show that $B$ computes a $g$ which represents $I$.

Let

$$
\begin{gathered}
f(e)= \begin{cases}0 & \text { if } T_{2 e} \text { was defined by the first case; } \\
\tau_{e}+1 & \text { otherwise. }\end{cases} \\
f^{-}(e)=0 \quad \text { if } f(e)=0 ; \quad f^{-}(e)=1 \text { otherwise. }
\end{gathered}
$$

We'll let $\delta \in \operatorname{Str}$ represent the hypothesis that $\delta \subset f^{-}$. Assuming this hypothesis, for $\operatorname{dom}(\delta)=n+1, B$ tries to recover $\left\langle T_{e}\right\rangle_{-1 \leq e \leq 2 n}$ and $A_{n}$. 
If $\delta \subset f^{-}$, eventually $B$ will have this right. If $\delta \not \subset f^{-}, B$ will not be so fortunate. Where $e$ is least so that $\delta(e) \neq f^{-}(e), e$ curses $\delta$ iff $f^{-}(e)=1$ and $\delta(e)=0 ; e$ disrupts $\delta$ iff $f^{-}(e)=0$ and $\delta(e)=1$. If $\delta$ is cursed, by assuming $\delta B$ eventually finds himself waiting eternally for a splitting which never comes; if $\delta$ is disrupted, constant changes in $B$ 's guesses at a node beyond which there are no splits will prevent $B$ 's guesses from settling down.

At each stage $s$, on hypohtesis $\delta B$ constructs the sequence of pre-trees $T_{e}^{\delta, s},-1 \leq e \leq 2 n$, as follows: $T_{-1}^{\delta, s}=\operatorname{Id} \uparrow \operatorname{Str}(s+1)$;

$$
T_{2 e}^{\delta, s}= \begin{cases}e-\operatorname{Split}_{s}\left(T_{2 e-1}^{\delta, s}\right) & \text { if } \delta(e)=0, \\ \operatorname{Full}\left(T_{2 e-1}^{\delta, s}, \tau_{e}^{\delta, s}\right) & \text { if } \delta(e)=1,\end{cases}
$$

where $\tau_{e}^{\delta, s}$ is the longest $\tau$ such that $e$-Split ${ }_{s}\left(T_{2 e-1}^{\delta, s}\right)(\tau)$ is defined, $\subset B$, and has no $e$-splitting on $T_{2 e-1}^{\delta, s}$ after $s$ steps of searching. Let $F(e, \delta, s)=$ $\operatorname{Coded}\left(B, T_{2 e}^{\delta, s}\right) . F(e, \delta, s)$ is $B$ 's stage $s$ guess at $A_{e} \uparrow k$, where $k=$ $\operatorname{dom}(F(e, \delta, s))$, based on hypothesis $\delta$.

$$
T_{2 e+1}^{\delta, s}=\operatorname{Code}\left(T_{2 e}^{\delta, s}, F(e, \delta, s)\right) .
$$

By remarks after the definitions of Code and Coded, this is well-defined.

Let $\operatorname{dom}(\delta)=n+1$. If $T_{2 n}^{\delta, s} \neq \varnothing$, for all $e$ with $-1 \leq e \leq 2 n, T_{e}^{\delta, s}$ $\neq \varnothing$; let $f^{\delta, s}: n+1 \rightarrow \omega$ be given by:

$$
f^{\delta, s}(e)= \begin{cases}0 & \text { if } \delta(e)=0 \\ \tau_{e}^{\delta, s}+1 & \text { if } \delta(e)=1\end{cases}
$$

$f^{\delta, s}$ is $B$ 's guess at $f \uparrow n+1$ at stage $s$, assuming $\delta$. If $T_{2 n}^{\delta, s}=\varnothing$, at stage $s$ $B$ hasn't enough information to make a guess. If $\delta \nsubseteq \delta^{\prime}, T_{e}^{\delta, s}=T_{e}^{\delta^{\prime}, s}$ for $e \leq 2 n$, and $f^{\delta, s}=f^{\delta^{\prime}, s} \uparrow n+1$.

We now consider the possible behavior of $f^{\delta, s}$ as $s$ increases.

(1) If $\delta \subset f^{-}$there is an $s$ such that for all $t \geq s, f^{\delta, t}$ is defined, $f^{\delta, t}=f^{\delta, s}=f \uparrow n+1, T_{e}^{\delta, t}=T_{e} \uparrow \operatorname{Str}\left(i_{t}^{t}\right)$ for $-1 \leq e \leq 2 n$, where $l_{e}^{t}$ is nondecreasing in $t$ and approaches $\omega$ for $t \geq s$; furthermore for $t \geq s$, $F(n, \delta, t) \subset A_{n}$, and so $\cup_{t \geq s} F(n, \delta, t)=A_{n}$. All this follows by induction on $n$.

(2) If $\delta$ is cursed, there is an $s$ such that either (a) for all $t \geq s, f^{\delta, t}$ is defined and $f^{\delta, t}=f^{\delta, s}$, or (b) for all $t \geq s, f^{\delta, t}$ is undefined. Furthermore, in case (a), for all $t \geq s, F(n, \delta, t)=F(n, \delta, s)$. To see this, suppose $e$ curses $\delta$; by (1) there is a stage $s_{0}$ by which $f^{\delta l e, t}$ is defined and equal to 
$f \uparrow e$ for all $t \geq s_{0}$; furthermore $T_{2 e-1}^{\delta, t}=T_{2 e-1} \uparrow \operatorname{Str}\left(l_{2 e-1}^{t}\right)$. Fix the least level $l$ such that for some $\delta$ with $\operatorname{dom}(\delta)=l, e-\operatorname{Split}\left(T_{2 e-1}\right)(\delta)$ is undefined. In building $T_{2 e}^{\delta, t}, B$ gets stuck at level $l$; so eventually $B$ is waiting for $e$-splittings on $T_{2 e^{-1}}^{\delta, t}$ of a string with no such $e$-splittings. So for some $s_{1} \geq s_{0}$, for all $t \geq s_{1}, \quad T_{2 e}^{\delta, t}=T_{2 e}^{\delta, s_{1}}$. Clearly for $-1 \leq j<j^{\prime} \leq 2 n$, $\operatorname{Range}\left(T_{j}^{\delta, t}\right) \subseteq \operatorname{Range}\left(T_{j^{\prime}}^{\delta, t}\right)$. So by induction we find $s$ so that for all $j \leq 2 n$ and $t \geq s, T_{j}^{\delta, t}=T_{j}^{\delta, s}$. If $T_{j}^{\delta, s}=\varnothing$, for $t \geq s, f^{\delta, t}$ is undefined. Otherwise $f^{\delta, t}(e)=0$.

(3) If $\delta$ is disrupted and $f^{\delta, s}$ is defined, for some $t>s$ either $f^{\delta, t}$ is undefined or $f^{\delta, t} \neq f^{\delta, s}$. To see this, suppose $e$ disrupts $\delta$ and select $s_{0}$ as above. Once $t \geq s_{0}, \tau_{e}^{\delta, t}$ goes to $\omega$ with $t$, since $e$-splittings for $e$ Split $_{t}\left(T_{2 e^{-1}-1}^{\delta, t}\right)\left(\tau_{e}^{\delta, t}\right)=e-\operatorname{Split}\left(T_{2 e-1}\right)\left(\tau_{e}^{\delta, t}\right)$ eventually turn up on $T_{2 e-1}$, and thus on $T_{2 e-1}^{\delta, t^{\prime}}$ for sufficiently large $t^{\prime} \geq t$; when this happens, $\tau_{e}^{\delta, t^{\prime}} \supseteq \tau_{e}^{\delta, t}$. Fixing $s$, for sufficiently large $t \geq s$, if $f^{\delta, t}$ is defined, $f^{\delta, t}(e)>f^{\delta, s}(e)$.

We now view $h \in \omega^{<\omega}$ as a guess at $f \uparrow \operatorname{dom}(h)$. Let $h^{-}(e)=0$ if $h(e)=0, h^{-}(e)=1$ otherwise. An $h$-block is a maximal interval $\left[s_{0}, s_{1}\right]$ $=\left\{t \mid s_{0} \leq t \leq s_{1}\right\}$ or $\left[s_{0}, \infty\right]=\left\{t \mid s_{0} \leq t\right\}$ such that for all $s$ in that interval, $h=f^{h^{-}, s}$. For any $h$ there are finitely many $h$-blocks. If $h^{-} \subset f^{-}$, this follows from (1); if $h^{-}$is cursed, this follows from (2). Note that if $h^{-} \subset f^{-}$or if $h^{-}$is cursed and (2a) is true, the final $h$-block is of the form $[s, \infty]$. If $h^{-}$is disrupted by $e$, this follows from (3) and the previous observation that for sufficiently large $t, \tau_{e}^{h^{-}, t}$ increases non-decreasingly with $t$. If $s$ and $t$ belong to one $h$-block and $s \leq t, F\left(e, h^{-}, s\right) \subset F\left(e, h^{-}, t\right)$ for $-1 \leq e<\operatorname{dom}(h)$. For the moment, assume that $\mathbf{a}_{0}=\mathbf{0}$. For $h \in \omega^{<\omega}$, $k \in \omega$ and $\operatorname{dom}(h)=n+1$, let

$$
(g)_{\langle h, k\rangle}(s)= \begin{cases}F\left(n, h^{-}, s\right)+1 & \text { if } s \text { belongs to the } k \text { th } h \text {-block; } \\ 0 & \text { otherwise. }\end{cases}
$$

Clearly $g \leq_{T} B$. If $h \not \subset f$, or if the $k$ th $h$-block is not of the form [s, ], $(g)_{\langle h, k\rangle}$ differs only finitely from $\lambda s$.0. If $h \subset f$ and the $k$ th $h$-block is of the form $[s, \infty]$, since $A_{n}=\cup_{t \geq s} F\left(n, h^{-}, t\right), A_{n} \leq_{T}(g)_{\langle h, k\rangle}$. Furthermore, $\lambda s . F\left(n, h^{-}, s\right) \leq_{T} A_{0} \oplus \cdots \oplus A_{n} \leq_{T} A_{n}$; thus $(g)_{\langle h, k\rangle} \leq_{T} A_{n}$. So either $\operatorname{deg}\left((g)_{\langle h, k\rangle}\right)=\mathbf{a}_{n}$ or $=\mathbf{0}=\mathbf{a}_{0}$. Thus $g$ represents $I$.

Now suppose $\mathbf{a}_{0} \neq \mathbf{0}$. Select $D \in \mathbf{a}_{0}$. Suppose we revised our definition of $(g)_{\langle h, k\rangle}(s)$ by requiring in the "otherwise" case that $(g)_{\langle h, k\rangle}(s)=$ $D(s)$. If $h^{-} \subset f^{-}$and the $k$ th block is of the form $\left[s_{0}, \infty\right]$, we still have $\operatorname{deg}\left((g)_{\langle h, k\rangle}\right)=\mathbf{a}_{n}$; if otherwise and if $h^{-}$is not cursed, $\operatorname{deg}\left((g)_{\langle h, k\rangle}\right)=$ $\mathbf{a}_{0}$. But if $h^{-}$is cursed and the $k$ th block is of the form $\left[s_{0}, \infty\right]$, 
$\operatorname{deg}\left((g)_{\langle h, k\rangle}\right)=\mathbf{0}$. To remedy this, we slightly hair-up the definition of $(g)_{\langle h, k\rangle}$ :

$$
\begin{gathered}
(g)_{\langle h, k\rangle}(2 s)= \begin{cases}F\left(h, h^{-}, s\right)+1 & \text { if } s \text { belongs to the } k \text { th } h \text {-block. } \\
D(s) & \text { otherwise }\end{cases} \\
(g)_{\langle h, k\rangle}(2 s+1)=D(s) .
\end{gathered}
$$

$g$ is now as desired.

COROLlARY. If I is a countable ideal, some minimal upper bound on I weakly represent $I$.

Proof. There is an $I^{\prime} \subseteq I$ cofinal in $I$ and linearly ordered; apply Theorem 1 to $I^{\prime}$ and notice that a minimal upper bound on $I^{\prime}$ is also one for $I$. bound?

Questions. Does every ideal have a representing minimal upper

Does a sequence $\left\langle a_{i}\right\rangle_{i<\omega}$ as above have a minimal upper bound which does not represent it?

The author thanks Richard Shore for fruitful discussions on matters related to the subject of this paper.

Received November 3, 1981 and in revised form July 23, 1982.

Deparment of Philosophy

CORNELL UNIVERSITY

ITHACA, NY 14853 



\section{PACIFIC JOURNAL OF MATHEMATICS EDITORS}

Donald BABBITT (Managing Editor)

University of California

Los Angeles, CA 90024

Hugo Rossi

University of Utah

Salt Lake City, UT 84112

C. C. Moore and Arthur Ogus

University of California

Berkeley, CA 94720
J. DugundiI

Department of Mathematics

University of Southern California

Los Angeles, CA 90089-1113

R. Finn and H. SAMELSON

Stanford University

Stanford, CA 94305

ASSOCIATE EDITORS
R. ARENS
E. F. BECKENBACH
B. H. NeUmanN
F. WOLF
K. YosHIDA (1906-1982)

\section{SUPPORTING INSTITUTIONS}

UNIVERSITY OF ARIZONA

UNIVERSITY OF BRITISH COLUMBIA

CALIFORNIA INSTITUTE OF TECHNOLOGY

UNIVERSITY OF CALIFORNIA

MONTANA STATE UNIVERSITY

UNIVERSITY OF NEVADA, RENO

NEW MEXICO STATE UNIVERSITY

OREGON STATE UNIVERSITY
UNIVERSITY OF OREGON

UNIVERSITY OF SOUTHERN CALIFORNIA

STANFORD UNIVERSITY

UNIVERSITY OF HAWAII

UNIVERSITY OF TOKYO

UNIVERSITY OF UTAH

WASHINGTON STATE UNIVERSITY

UNIVERSITY OF WASHINGTON 


\section{Pacific Journal of Mathematics}

\section{Vol. 108, No. $1 \quad$ March, 1983}

Waleed A. Al-Salam and A. Verma, $q$-Konhauser polynomials $\ldots \ldots \ldots \ldots 1$

Alfred David Andrew, The Banach space JT is primary $\ldots \ldots \ldots \ldots \ldots . . .6$

Thomas E. Bengtson, Bessel functions on $P_{n} \ldots \ldots \ldots \ldots \ldots \ldots$

Joaquim Bruna Floris and Francesc Tugores, Free interpolation for

holomorphic functions regular to the boundary $\ldots \ldots \ldots \ldots \ldots \ldots \ldots \ldots$

Peter Dierolf and Susanne Dierolf, Topological properties of the dual pair

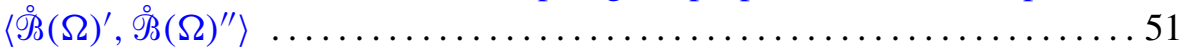

Gerald Arthur Edgar, An ordering for the Banach spaces $\ldots \ldots \ldots \ldots \ldots 83$

Basil Gordon, A proof of the Bender-Knuth conjecture . . . . . . . . . . . . . 99

Harold T. Hodes, A minimal upper bound on a sequence of Turing degrees

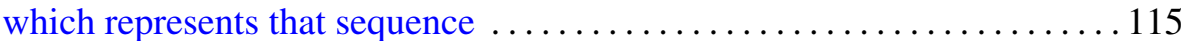

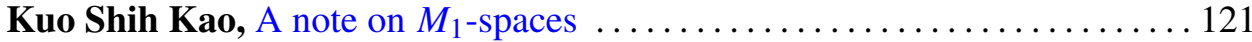

Frank Kost, Topological extensions of product spaces ................ 129

Eva Lowen-Colebunders, On the convergence of closed and compact

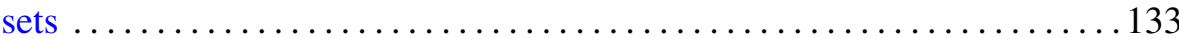

Doron Lubinsky, Divergence of complex rational approximations . . . . . . 141

Warren May and Elias Hanna Toubassi, Endomorphisms of rank one

mixed modules over discrete valuation rings $\ldots \ldots \ldots \ldots \ldots \ldots \ldots \ldots \ldots$

Richard Patrick Morton, The quadratic number fields with cyclic

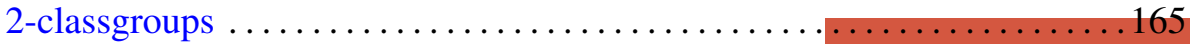

Roderic Murufas, Rank of positive matrix measures . . . . . . . . . . . . 177

Helga Schirmer, Fixed point sets of homotopies . . . . . . . . . . . . . 191

E. Taflin, Analytic linearization of the Korteweg-de Vries equation ........ 203

James Thomas Vance, Jr., $L^{p}$-boundedness of the multiple Hilbert

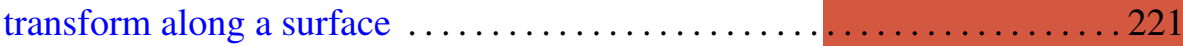

Hiroshi Yamaguchi, A property of some Fourier-Stieltjes transforms . . . . . 243 